\title{
Correction: The dual PI3K/mTOR inhibitor dactolisib elicits anti- tumor activity in vitro and in vivo
}

\author{
Fei Shi ${ }^{1, *}$, Jinying Zhang ${ }^{2, *}$, Hongyu Liu ${ }^{3}$, Liangliang $\mathbf{W u}^{3}{ }^{3}$, Hongyu Jiang ${ }^{4}$, Qiyan \\ $\mathbf{W u}^{3}$, Tianyi Liu ${ }^{3}$, Meiqing Lou ${ }^{1, \#}$ and Hao $\mathbf{W u}^{5, \#}$ \\ ${ }^{1}$ Department of Neurosurgery, Shanghai General Hospital, Shanghai Jiao Tong University School of Medicine, Shanghai \\ 200000, China \\ 2 Institute of Basic Medicine Science, Chinese PLA General Hospital, Beijing 100853, China \\ ${ }^{3}$ Key Laboratory of Cancer Center, Chinese PLA General Hospital, Beijing 100853, China \\ ${ }^{4}$ Department of Anesthesiology, Wuxi Third People's Hospital, Wuxi, Jiangsu 214000, China \\ ${ }^{5}$ Department of Neurosurgery, Xuanwu Hospital, Capital Medical University, Beijing 100053, China \\ * These authors contributed equally to this work \\ \# Co-senior authors \\ Correspondence to: Hao Wu, email: haowv017@126.com \\ Meiqing Lou, email: loumqdoc@126.com \\ Published: March 30, 2018
}

Copyright: Shi et al. This is an open-access article distributed under the terms of the Creative Commons Attribution License 3.0 (CC BY 3.0), which permits unrestricted use, distribution, and reproduction in any medium, provided the original author and source are credited.

This article has been corrected: Dr. Meiqing Lou is included as a co-corresponding author.

Correspondence to: Hao Wu, email: haowu017@126.com

Meiqing Lou, email: loumadoc@126.com

Original article: Oncotarget. 2018; 9:706-717. https://doi.org/10.18632/oncotarget.23091 